\title{
Correction to: Elastic superhydrophobic and water glass-based silica aerogels and applications
}

\author{
Amirisetty Venkateswara Rao ${ }^{1}$
}

Published online: 15 February 2019

(c) Springer Science+Business Media, LLC, part of Springer Nature 2019

\section{Correction to: Journal of Sol-Gel Science and Technology 2018 https://doi.org/10.1007/s10971-018-4825-5. Published online 19 Oct 2018.}

In the original version of this article unfortunately contained mistakes. The errors have been corrected in this correction.

Page 1, Comma, after 416004.

Page 3, Table 1, reference numbers: 31 instead of 26; 103 instead of 28; 104 instead of 29; 30 instead of $25 ; 32$ instead of 27; 67, 68 instead of 30,31; 69 instead of 32; Ambient Pressure Drying (APD) instead of Ambient Pressure Drying, 46, 52 instead of 33,34; 58 instead of 35.

Left, from bottom, 2nd line after [32], full stop. Right from bottom, 3rd line, aerogels by Ambient Pressure Drying (APD) instead of aerogels by APD.

Page 5, Caption of Fig. 2, first line, aerogel instead of aereogel.

Page 6, Fig. 4, b: Please put the white curve for angle Theta like in the other two figures, but white curve for the angle instead of black curve to see in a better way. Fig. 4, caption, small b, instead of B; and the reference should be [45] instead of [43]. 63].

Page 8, Fig. 6, caption references; $[63,64]$ instead of $[62$,

Page 9, Fig. 8 , in caption, the reference, $[46,52]$ instead of [52].

The original article can be found online at https://doi.org/10.1007/ s10971-018-4825-5.

Amirisetty Venkateswara Rao

avrao2012@gmail.com

1 Air Glass Laboratory, Department of Physics, Shivaji University, Kolhapur 416004 Maharashtra State, India
Page 10, Right column, line 4, from top, for Si left side three bonds instead of two bonds aprrox.sign. Right column, first paragraph end line, reference should be [65] instead of [63].

Page 11, Right column, line 8, reference should be [69] instead of [71]

Page 12, Left column, line 4, silylated instead of silylateed, Fig. 10, in caption, the reference should be [70] instead of [68].

Page 13, Right Column, line 15, nonsilylated instead of nonsillylated, Right column, 2nd paragraph, line 7, the reference should be [75] instead of [73]; Right side from bottom, line 9, the reference should be [74, 75] instead of [73]; In Fig.11, caption, the reference should be [71] instead of [73]

Page 14, Fig.12, caption end, reference [71] should be added. Left column, line10, the reference should be [69] instead of [71]; Left first paragraph, from end 2nd line, reference [78] should be deleted. Then, next line instead of only refernce[76] it should be $[76,106]$

Page 15, Left column, third equation, it should be Refractive index instead of Refraction index.

Page 16, Fig. 14 caption, it should be [58] instead of [56]. Left column, line 4, it should be [78-81] instead of [78, 81]; Right side column, it should be [46, 58, 63, 70] instead of $[46,63,70]$.

Page 17, Right column, third paragraph, [58] instead of [52]; and 4 lines below, it should be monolithicity instead of nonolithicity.

Page 18, Right column, line 5 it should be systems [Fig. 16]. instead of systems.

Page 20, Right column, line 6, Reference should be [58] instead of [56]; line 4 from bottom, silica aerogel instead of silica baerogel.; in the same line, add, 'with' after comparison.

Page 21, Fig. 21, Please cut some top portion so that the word 'Diesel ' can be seen.

Page 22, Left column, line 2, it should be), laser beam which has the instead of), which is the. 
Page 23, Right column, 2nd paragraph, from bottom line 5, it should be [58] instead of [56]. Section 9: Conclusions, line 5, after solids, add the reference [105] after solids.

Page 25, Left column, paragraph 3 , from bottom, line 6 , it should be 1.001 instead of 0.001

Page 26, In reference 35, there should be spacing between "spill clean"
Page 27, in Reference 105, after (eds) Aerogels : A novel class of

In Table 1 , item 8,2 nd line, it should be (APD) without solvent exchange., instead of, (APD) solvent exchange.

The original article has been corrected. 